\title{
A Calculable, Transportable Audio-Frequency AC Reference Standard
}

\author{
NILE M. OLDHAM, MEMBER, IEEE, PAUL S. HETRICK, AND XIANGREN ZENG
}

\begin{abstract}
A transportable ac voltage source is described, in which sinusoidal signals are synthesized digitally in the audio-frequency range. The rms value of the output waveform may be calculated by measuring the de level of the individual steps used to generate the waveform. The uncertainty of this calculation at the 7-V level is typically less than $\pm 5 \mathrm{ppm}$ from $60 \mathrm{~Hz}$ to $2 \mathrm{kHz}$ and less than $\pm 10 \mathrm{ppm}$ from $30 \mathrm{~Hz}$ to $15 \mathrm{kHz}$.
\end{abstract}

\section{INTRODUCTION}

$\mathrm{D}^{1}$ IGITALLY SYNTHESIZED sine wave sources have become commonplace in metrology laboratories in recent years, particularly where a precise knowledge of the phase angle between two waveforms is required. Impedance and power bridges [1], [2] as well as phase angle standards [3] rely on waveform synthesis techniques to achieve state-of-the-art accuracy. The approach has been applied to ac voltage standards [4], [5] with some success. However, imperfections in the steps used to generate the waveforms have limited the ability to calculate the rms value of the ac signal based on a knowledge of the static voltage of each step. Transient energy due to waveform aberrations at the step transitions (often referred to as "glitches") adds to the signal power making it difficult to predict the rms value accurately.

\section{Generator Circuit}

A "deglitching" technique, to improve phase angle precision [6], has been employed to enhance the step quality in an audio-frequency, digitally synthesized source (DSS). Charge-coupled and switching glitches, as well as current settling time in high-resolution digital-to-analog converters (DAC's), may be reduced by toggling between two converters which are updated at different times. A simplified diagram of the DSS is shown in Fig. 1. Digital values of the waveform, stored in a read-only-memory (ROM) are sequentially latched into multiplying DAC's, MDAC1 and MDAC2, on the positive transition of clock signals $A$ and $B$, respectively. Fast CMOS switches $\left(S_{1}\right.$, $S_{2}, S_{3}, S_{4}$ ) steer the output current from each MDAC to ground until the current step has settled, and then steer the current into the summing node of a wide-band operational amplifier. At time $t_{1}$ new data are latched (Lat1)

Manuscript received June 10, 1988.

N. M. Oldham and P. S. Hetrick are with the Electrosystems Division, National Bureau of Standards, Gaithersburg, MD 20899.

$X$. Zeng is with the Department of Electrical Engineering, Chongqing University, Chongqing, China.

IEEE Log Number 8825617.

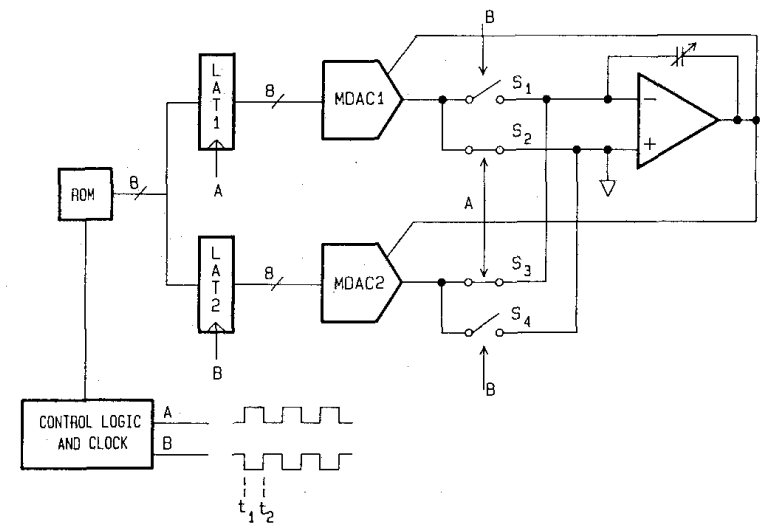

Fig. 1. Simplified diagram of the dual-DAC, digitally synthesized source.

into MDAC1, $S_{2}$ and $S_{3}$ are closed, and $S_{1}$ and $S_{4}$ are opened. While MDAC1 is settling, MDAC2 supplies the output step, and thus most of the transient energy generated by the MDAC1 is dissipated in the ground plane. At time $t_{2}$, new data are latched (Lat2) into MDAC2, $S_{1}$ and $S_{4}$ are closed, and $S_{2}$ and $S_{3}$ are opened. MDAC1 now supplies the output step while MDAC2 is settling. Each MDAC supplies half of the steps and thus operates at half of the clock rate, allowing the generator to operate at higher clock rates than would be possible with a single DAC.

The feedback path, from the amplifier output back to its summing input, is determined by the MDAC that is connected to the summing input. In addition to the gain accuracy and temperature tracking realized by using the internal feedback resistor in each MDAC, this approach minimizes the effects of switch contact-resistance (inside the feedback loop the switch resistance is divided by the amplifier loop gain).

An oscilloscope trace of a portion of a 128-step sine wave approximation generated by a single DAC with a clock rate of $1 \mathrm{MHz}$ is shown in Fig. 2(a). The corresponding waveform, generated by toggling two DAC's into the same output stage, is shown in Fig. 2(b). The major transition glitch as well as other structural differences between the steps in the single DAC system are virtually eliminated with the two DAC approach.

While the waveform in Fig. 2(b) is noticeably improved, the flatness of its rms value versus frequency is not well defined (at the parts per million level) above about 


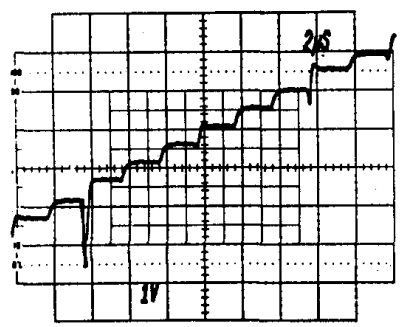

(a)

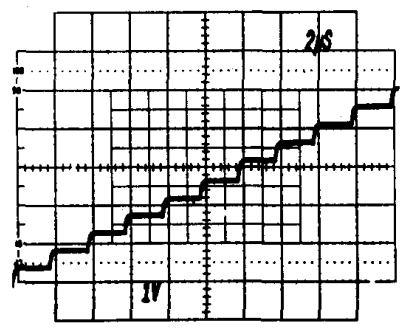

(b)

Fig. 2. Oscilloscope traces of an unfiltered portion of a 128-step digitally synthesized sine wave (1-MHz clock rate). (a) Using a single DAC. (b) Using the dual-DAC deglitching circuit.

$2 \mathrm{kHz}$. The deglitching technique does not eliminate the effects of charge injection from the logic signals into the switches, timing skews in the switches, and in the control signals, or amplifier errors (including overshoot, ringing, and slew limiting). In the frequency band from 2 to 15 $\mathrm{kHz}$ these effects tend to increase the rms value as the frequency is increased. By sacrificing frequency response, the amplifier can be band limited to flatten the rms gain to within $\pm 10 \mathrm{ppm}$, well into the audio-frequency range. This trimming is done using a small variable capacitor which shunts the feedback circuit of the output amplifier.

A prototype calculable DSS has been constructed using 18-bit MDAC's. For simplicity only the most significant 8 bits are used to synthesize the waveform. 18-bit converters were selected to obtain the component stability and low temperature coefficients that are inherent in high-resolution DAC's.

The DSS is normally operated using the internal $10-\mathrm{V}$ $\mathrm{dc}$ reference in one of the MDAC's as a reference voltage for both MDAC's. The 10-V level produces a sine wave with an rms value of approximately $7 \mathrm{~V}$. Any stable reference between 0 - and $10-\mathrm{V}$ dc may be supplied externally to produce sine waves with rms values between 0 and $7 \mathrm{~V}$; however, lower reference voltages seriously degrade the performance.

An internal clock pulse, the rate of which may be selected in 16 binary increments, is used to generate 128 step sine wave approximations between $1 \mathrm{~Hz}-15 \mathrm{kHz}$. However, the DSS may be operated using an external clock in order to obtain better frequency precision. An external clock is also used in the step calibration mode described below.

\section{RMS Value From a Step Calibration}

The rms voltage generated by the DSS is computed by stepping through the ROM at low speed to measure the amplitude of each step with a high-accuracy dc digital voltmeter (DVM). The rms value of the generated waveform using this "step calibration" is determined by computing the square root of the mean value of the square of each of the measured step voltages

$$
V_{\mathrm{rms}}=\left(\sum_{i=1}^{N}\left[v_{i}(1+C)\right]^{2} / N\right)^{1 / 2}
$$

where

$v_{i}$ voltage of the $i$ th step,

$N$ number of steps in one period,

$C$ DVM correction.

The uncertainty of the step calibration is a function of the gain and linearity errors of the DVM used to measure each step. To minimize gain errors, the DVM is calibrated immediately prior to the step calibration. The shortterm stability of this calibration is critical, and is typically within \pm 1 ppm during the test. The DVM linearity is also important since the computed rms value is based on measurements made at many different voltage levels. The results reported in this paper were obtained using a multislope integrating DVM with nonlinearities of less than \pm 2 ppm of full scale. The step calibration is automated by connecting the "conversion complete" digital output from the DVM to the external clock input of the DSS. The DVM is connected to a desktop computer via the IEEE488 bus and a BASIC software routine computes the rms value. The short-term repeatability of the rms value computed by the step calibration is typically $\pm 1 \mathrm{ppm}$.

A simplified step calibration, consisting of "offset" and "gain" measurements made at plus and minus full scale, has been used with linear single DAC generators [5], [7]. However, the nonlinearity of the dual DAC generator described in this paper can be quite large (a function of linearity, offset, and gain differences between the two DAC's), therefore, the complete step calibration is required. The best multislope integrating DVM's are typically an order of magnitude more linear then the best multiplying DAC's and the complete step calibration should enhance the accuracy of single DAC generators as well.

\section{BANDwidTh Considerations}

The concept of a "calculable source," which relies on measurements of the dc level of each step, requires the waveform to have the same shape at all frequencies of interest. The rms value may be computed by (1) only if the staircase shape is not distorted by dynamic errors in the generation circuit or post filtering (a standard procedure for removing sampling harmonics). Under ideal conditions, it can be shown that the rms value of the reconstructed sine wave is identical to the pure sinusoid from which the samples were obtained [8].

When a sinusoidal signal is approximated using digital 


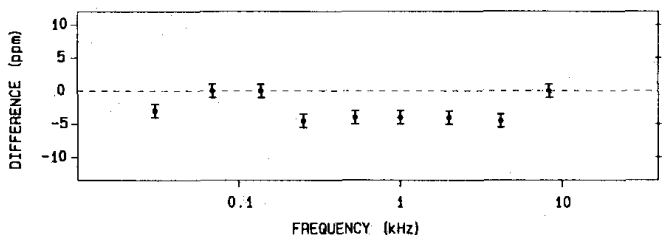

(a)

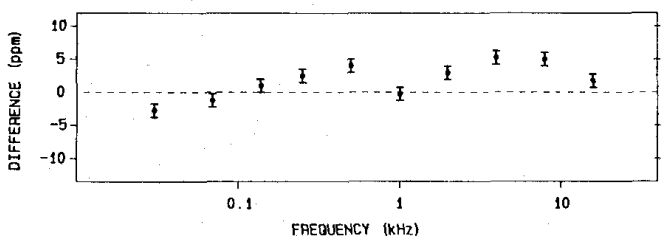

(c)

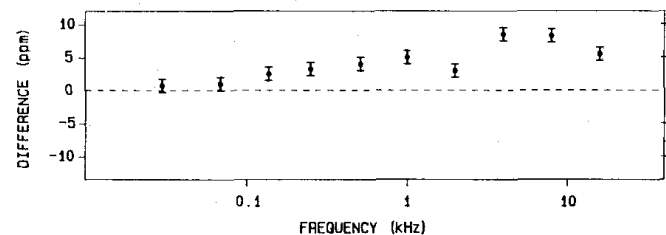

(b)

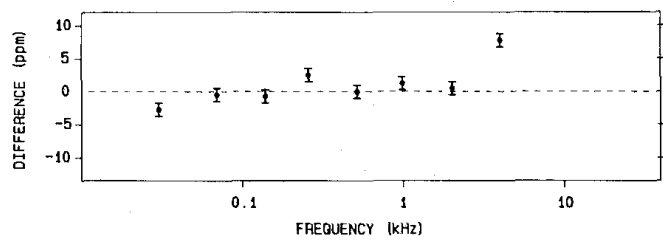

(d)

Fig. 3. The difference in parts per million between the rms value computed by the step calibration (dashed line) and the rms value measured using thermal techniques: (a) DSS1 using the internal clock to generate a sinusoidal waveform. (b) DSS2 using the internal clock to generate a sinusoidal waveform. (c) DSS2 using an external clock to generate a sinusoidal waveform. (d) DSS2 using an external clock to generate a nonsinusoidal waveform.

synthesis, the fundamental frequency is mixed with the clock frequency to produce sampling harmonics. These occur as sum and difference pairs around harmonics of the clock frequency. For a 128-step sine wave the first four sampling harmonics occur at the 127th, 129th, 255th, and 257 th harmonic of the fundamental. In a perfect system there are an infinite number of sampling harmonics, the amplitudes of which are defined by

$$
V_{i}=V \sin \left(\pi F_{i} T\right) /\left(\pi F_{i} T\right)
$$

where

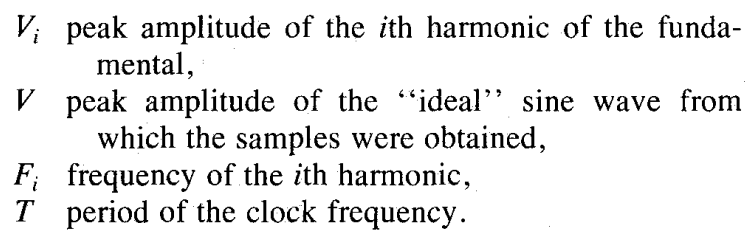

The amplitude of the fundamental component $\left(F_{1}\right)$ of a reconstructed sine wave is also defined by (2); and for a 128 -step sine wave, $V_{i}=0.999900 \mathrm{~V}$. The sampling harmonics thus deliver only $100 \mathrm{ppm}$ of the signal power (rms value), and it can be shown that less than $3 \mathrm{ppm}$ of the signal power is delivered above the 1025th harmonic of the fundamental. In practical terms, this means that an rms voltmeter with a bandwidth in excess of $1 \mathrm{MHz}$ is required to measure an unfiltered, $1-\mathrm{kHz}$ synthesized sine wave to an uncertainty of $\pm 3 \mathrm{ppm}$. In the DSS, frequency components above $10-20 \mathrm{MHz}$ are eliminated by the feedback capacitor used to trim the rms response.

Additional distortion is caused by the quantization of each of the steps. The normalized magnitude of the quantization level is given by $q=1 / 2^{n}$, where $n$ is the number of effective bits in the DAC. Since only the most significant 8 bits are used in the DSS, $q=1 / 256$. For 8 -bit quantization, the noise power $\left(P_{n}=q^{2} / 12\right)$ contributes less than $\pm 1 \mathrm{ppm}$ to the signal power [8].

\section{RESUlts}

The rms value, computed using the step calibration, has been compared to the thermally measured value at sine wave frequencies between $30 \mathrm{~Hz}$ and $15 \mathrm{kHz}$. Thermal measurements, which represent the state-of-the-art, were made using an automatic voltage calibration system [9] based on coaxial thermal voltage converters (TVC's). This system is capable of measuring all of the harmonic power since its bandwidth is in excess of $100 \mathrm{MHz}$. Uncertainties associated with this measurement system are:

1) ac-dc difference of the TVC- $\pm 2 \mathrm{ppm}$;

2) lumped uncertainties due to the rest of the system$\pm 2 \mathrm{ppm}$;

3) the standard deviation of the mean of 15 observations- \pm 1 ppm.

To minimize dc uncertainties, the DVM used in the step calibration is calibrated by the dc source used as a reference for the thermal measurements. This source is thus considered the $\mathrm{dc}$ reference for both measurements. While its short-term stability is critical, its absolute accuracy is not.

The estimated standard deviations of the step and thermal calibrations were computed in accordance with [10] by

$$
U=\left(a^{2}+\left(\sum b_{i}^{2}\right) / 3\right)^{1 / 2}
$$

where

$a$ standard deviation of the observations,

$b_{i}$ limits of the estimated errors. 
The resultant standard deviations are $1.6 \mathrm{ppm}$ for the step calibration and $1.9 \mathrm{ppm}$ for the thermal measurement.

Results of step and thermal measurements of the DSS are shown in Fig. 3. To demonstrate the performance of different units under various operating conditions, two sources, DSS1 and DSS2, were measured using both internal and external clocks to synthesize 128-step sine wave approximations (Fig. 3(a)-3(c)). As a further demonstration, the results of measurements on a nonsinusoidal waveform (sine wave approximation with 30 -percent third harmonic) are shown in Fig. 3(d). The plots show the average difference between three sets of measurements consisting of two step calibrations (represented by the dashed line) and 5 thermal measurements (plotted points with one-standard deviation error bars) at each frequency.

Agreement between the two methods is better than \pm 5 ppm from $30 \mathrm{~Hz}$ to about $2 \mathrm{kHz}$. Above $2 \mathrm{kHz}$ the rms value of the DSS is less predictable, becoming increasingly dependent on the bandwidth adjustment described above. It was, however, possible to trim the rms value of DSS2 between $30 \mathrm{~Hz}$ and $15 \mathrm{kHz}$ to within $\pm 10 \mathrm{ppm}$ of the calculated value. This "flatness" adjustment appears to be very stable versus time and has proven to be relatively immune to normal mechanical shocks encountered during shipment.

\section{Conclusions}

The rms value of a digitally synthesized waveform may be estimated by measuring the dc level of each of the steps used to generate the waveform. The accuracy of this estimate depends on the difference between the static and dynamic performance of the DAC. To minimize DAC glitches due to charge injection and switching skews, a deglitching circuit which uses a fast switch to toggle between two DAC's is utilized. This circuit substantially improves the step quality while increasing the effective frequency range of the source.

The calculated rms value agrees with the thermally measured value to within $\pm 5 \mathrm{ppm}$ from $30 \mathrm{~Hz}$ to $2 \mathrm{kHz}$. In this frequency range the digital-synthesis/calculation technique reenforces the validity of the traditional thermal approaches and may be considered as an independent dcac transfer standard. At higher frequencies, the DSS is increasingly dependent on components, but it may be pre- cisely adjusted so that the flatness of its rms value versus frequency is within $\pm 10 \mathrm{ppm}$ out to $15 \mathrm{kHz}$. With faster DAC's it should be possible to extend this performance to higher frequencies.

Four prototype digitally synthesized sources have been developed and are being shipped to metrology laboratories in the U.S. in order to evaluate their usefulness as ac transport standards. The sources have been used to verify the accuracy of thermally-based voltage measurement systems, to calibrate high-accuracy ac DVM's, and to characterize the ac-dc difference of thermal voltage converters (particularly at frequencies below $20 \mathrm{~Hz}$ where the DSS is more accurate than most thermal techniques).

\section{ACKNOWLEDGMENT}

The authors would like to thank R. Palm, O. Laug, and B. Waltrip for their contributions to this paper. The concept of measuring the individual steps of a synthesized waveform (off-line) to compute the rms value was proposed in 1978 by J. R. Kinard.

\section{REFERENCES}

[1] H. Bachmair and R. Vollmert, "Comparison of admittances by means of a digital double-sinewave generator," IEEE Trans. Instrum. Meas., vol. IM-29, pp. 370-372, Dec. 1980

[2] N. M. Oldham and O. Petersons, "Calibration of standard wattmeters using a capacitance bridge and digital generator," IEEE Trans. Instrum. Meas., vol. IM-34, pp. 521-524, Dec. 1985 ,

[3] R. S. Turgel and N. M. Oldham, "High-precision audio-frequency phase calibration standard," IEEE Trans. Instrum. Meas., vol. 27, pp. $460-463$, Dec. 1978 .

[4] J. R. Kinard and L. A. Harris, "A digitally synthesized sine wave source with $\pm 1 \mathrm{ppm}$ amplitude stability," in Euromeas '77 Conf. Digest, Institute Elect. Eng. Conf. Pub. no. 152, Sept. 1977.

[5] N. M. Oldham, "A 50-ppm ac reference standard which spans 10 Hz-50 kHz," IEEE Trans. Instrum. Meas., vol. IM-32, pp. 176179, Mar. 1983.

[6] D. T. Hess and K. K. Clarke, "Circuit techniques for use in a digital phase-angle generator," IEEE Trans. Instrum. Meas., vol. IM-36, pp. 394-399, June 1987.

[7] P. N. Milianic, "Calibrator for alternating voltage, current and power," in CPEM'88 Dig., Proc. Conf. Precision Electromagn. Meas. Tsukuba, Japan, pp. 345-346, 1988.

[8] N. M. Oldham, "Digital waveform synthesis techniques," NBS Special Publ. 707, pp. 1-13, Oct. 1985.

[9] N. M. Oldham, M. E. Parker, A. M. Young, and A. G. Smith, "A high accuracy $10 \mathrm{~Hz}-1 \mathrm{MHz}$ automatic ac voltage calibration system," IEEE Trans. Instrum. Meas., vol. IM-36, pp. 883-887, Dec. 1987.

[10] BIPM, "Working group on the statement of uncertainties," Metrologia, vol. 17 , no. 2, p. 73 . 